\title{
Study of ${ }^{123} \mathrm{Ag} \beta$-decay at ALTO
}

\author{
Dmitry A. Testov ${ }^{1, \mathrm{a}} \mathbb{D}$, A. P. Severyukhin ${ }^{1}$, B. Roussière ${ }^{2}$, N. Arsenyev ${ }^{1}$, F. Ibrahim ${ }^{2}$, M. Lebois ${ }^{2}$, I. Matea ${ }^{2}$, \\ Yu. Penionzhkevich ${ }^{1,3}$, V. Smirnov ${ }^{1}$, E. Sokol ${ }^{1}$, I. Stefan ${ }^{2}$, D. Susuki ${ }^{2}$, D. Verney ${ }^{2}$, Jh. Wilson ${ }^{2}$ \\ ${ }^{1}$ Joint Institute for Nuclear Research, Joliot-Curie 6, Dubna, Moscow Region, Russia 141980 \\ ${ }^{2}$ Université Paris-Saclay, CNRS/IN2P3, IJCLab, Orsay, France \\ ${ }^{3}$ National Research Nuclear University, Kashirskoye shosse 31, Moscow, Russia 115409
}

Received: 27 October 2020 / Accepted: 19 January 2021 / Published online: 15 February 2021

(c) The Author(s), under exclusive licence to Società Italiana di Fisica and Springer-Verlag GmbH Germany, part of Springer Nature 2021

Communicated by Anu Kankainen

\begin{abstract}
The neutron rich ${ }^{123} \mathrm{Ag}$ nucleus was populated via induced photofission of $\mathrm{UC}_{x}$ at the ALTO ISOL facility. Its $\beta$-decay properties were studied by means of detecting $\beta$-delayed $\gamma$ and neutron activities. The measured half-life $\mathrm{T}_{1 / 2}=0.350(20) \mathrm{s}$ agreed with the earlier data; the $\beta$-delayed neutron emission branching $\mathrm{P}_{1 n}=1.01(24) \%$ was reestablished using $\gamma$ and neutron counting.
\end{abstract}

\section{Introduction}

The $\beta$-delayed neutron emission probability $\left(P_{n}\right)$ and the half-life are the two key ingredients of $\beta$-decay that depend on the integrated quantities of the nuclear structure. The halflife provides information on the integrated total $\beta$-strength function, whereas $P_{n}$ value probes the part of the strength function above the neutron separation energy $\left(S_{n}\right)$. Therefore, measurements of these values in experiments often provides the first insight into the nuclear structure. Although the mechanism of $\beta$-decay of nuclei is in principle understood, development of a satisfactory description coherent with new data sets for neutron-rich species is highly required. A significant experimental effort is currently underway to reveal the role of neutron excess in the shell evolution [1]. The current experimental studies of $\beta$-delayed (multi) neutron emission in the regions of the chart of nuclides, which are important from the astrophysical point of view, are facing a new era: pure beams of neutron-rich nuclei of hard-to-reach mass regions of ${ }^{78} \mathrm{Ni}$ and ${ }^{132} \mathrm{Sn}$, have become available at radioactive ion beam facilities [2].

Reliable knowledge of the nuclear physics properties for the extremely neutron-rich nuclei along the r-process path is needed to interpret the observational data in the framework of proposed astrophysical models [3-5]. As the neutron excess increases near the drip line, we expect the properties of the giant resonances and of the low-lying collective states to be dramatically affected at extreme $N / Z$ ratios [6]. The formation of the neutron-skin may introduce a new type of collective mode in neutron-rich nuclei corresponding to the out-of-phase vibration of the skin against the neutron-proton inert core [7]. The concentration of the electric dipole $(E 1)$ strength around the particle separation energy is commonly called the pygmy dipole resonance (PDR). The occurrence of non-negligible low-lying $E 1$-strength can influence the radiative neutron capture cross section by orders of magnitude and, consequently, also the rate of the astrophysical $r$-process nucleosynthesis (see details in Ref. [8]). In case of the neutron-rich nuclei in the vicinity of $A \approx 130$, the $E 1$ strength could be significantly more important than in nuclei close to the valley of $\beta$-stability.

In this context, the $\beta$-decay properties of heavy $\mathrm{Ag}$ and $\mathrm{Cd}$ isotopes are ideal candidates for such studies. Particularly, in very neutron-rich $\mathrm{Ag}$ nuclei the total energy released through $\beta$-decay, $Q_{\beta}$, can go beyond $10 \mathrm{MeV}$ (in case of ${ }^{130} \mathrm{Ag}$, for example, $Q_{\beta}=15.42(30) \mathrm{MeV}$ [9]) and subsequently even deeply bound neutrons can decay into protons. When the $\mathrm{Cd}$ daughter nucleus is produced in a high-energy configuration above the neutron separation energy $\left(S_{n}\right)$, it usually de-excites through the neutron emission. On the other hand, the rearrangement of nuclear matter could proceed through collective modes of de-excitation in the daughter isotope, involving also the most superficial nucleons. Namely, microscopic calculations by Refs. $[10,11]$ showed that the GamowTeller (GT) transitions of deeply-bound neutrons can trigger coherent dipolar oscillations (like the PDR), which in turn engender a significant emission of $E 1 \gamma$-radiation. It is worth mentioning that the inclusion of the coupling terms with more complex configurations than $1 p-1 h$ ones sub-

\footnotetext{
a e-mail: dumon@jinr.ru (corresponding author)
} 
stantially enriches the low-energy spectrum of nuclei in the $A \approx 130$ mass range, see, e.g., Ref. [12].

The r-process abundance region around $A \approx 110$, prior to the $A=130$ peak, is one of the regions of intense interest, where the astrophysical models underestimate the abundances by an order of magnitude or more [13]. The newly measured $P_{n}$ values in this region will directly affect the final r-process abundance pattern through the $\mathrm{A}=114-120$ to the onset $(\mathrm{A}=\approx 123)$ of the $\mathrm{A}=130$ peak region.

The $\beta$-delayed neutron emission from ${ }^{123} \mathrm{Ag}$ has been reported previously from the OSIRIS mass-separator in Refs. $[14,15]$. The mass separated heavy fission products have been studied using a neutron counter comprised of $30{ }^{3} \mathrm{He}$ tubes. The weak neutron activities seen at the 122 and 123 masses were assigned to the silver isotopes. Consequently, due to 390(30) ms half-life measured for the 123 mass the neutron activity (still weak) was assigned to decay of ${ }^{123} \mathrm{Ag}$. Later at the TRISTAN on-line isotope separator facility at Brookhaven National Laboratory, the half-lifes and $P_{n}$ values have been measured for ${ }^{121-124} \mathrm{Ag}$ [16]. In a single set of experiments, using mass-separated fission fragments, the neutron and $\beta$ growth (decay) curves were measured simultaneously at masses 120-124 and at 127. The neutron decay curve resulted in a 300(10) ms half-life which was assigned to decay of ${ }^{123} \mathrm{Ag}$. The $P_{n}$ value of $0.55(9) \%$ was determined as a ratio of the neutron to $\beta$ saturation activities, corrected by the corresponding efficiencies. Neutrons were straight detected by $40{ }^{3} \mathrm{He}$ proportional tubes embedded in polyethylene. The more recent measurements of the $\beta$-delayed neutron emission branching for neutron-rich $\mathrm{Ag}$ isotopes, populated in a fragmentation reaction, have been done at the National Superconducting Cyclotron Laboratory at Michigan State University [17]. The yielded half-life of 272(24) ms was derived using time difference between the ion implantation and the correlated $\beta$-decay. The half-life was slightly shorter than reported previously $[15,16]$. The $P_{n}$ value was determined as $1.0(5) \%$ based on the number of $\beta$-neutron coincidence events divided by the number of $\beta$ decays following the implantation of ${ }^{123} \mathrm{Ag}$ heavy ions. Neutrons were detected by the NERO array consisting of $60{ }^{3} \mathrm{He}$ and $\mathrm{BF}_{3}$ counters.

Regardless the numerous studies the ${ }^{123} \mathrm{Cd}$ level scheme populated in $\beta$-decay of ${ }^{123} \mathrm{Ag}$ was only reported in the study performed at the Buenos Aires Tandem accelerator facility [18]. The ${ }^{123} \mathrm{Ag}$ nuclei was produced as a massseparated fission product from the fast-neutron fission of natural U. Then $\gamma$-spectrometry measurements were performed using two Ge(HP) detectors. Spins and parities for the most important states were suggested upon $\log (\mathrm{ft})$ values obtained from measured $\gamma$-ray transition intensities.

In the work presented here the neutron-rich ${ }^{123} \mathrm{Ag}$ nucleus was revisited to study for the first time its $\beta$-decay by simultaneous detection of $\beta$-delayed neutron- and $\gamma$ activities. The heavy Ag nuclei were populated using induced photofission of uranium at the ALTO ISOL facility $[19,20]$ and measured at the BEDO $\beta$-decay tape station [21] equipped with the TETRA neutron detector containing 80 proportional counters of ${ }^{3} \mathrm{He}$ [22]. Up to now at ALTO the studies were concentrated in the region of heavy Ga nuclei crossing the $N=50$ neutron closed shell: competition of the neutron emission process with the emission of high energy $\gamma$-rays [10]; population in $\beta$-decay resonance low-energy structures [23]. In the present paper we report the first measurement of a $\beta$ delayed neutron precursor in the ${ }^{132} \mathrm{Sn}$ region performed at ALTO.

\section{Experimental details}

Neutron-rich isotopes were produced via induced photofission at ISOL facility ALTO $[19,20]$. A $50 \mathrm{MeV}, \sim 7 \mu \mathrm{A}$ electron beam was applied to $\mathrm{UCC}_{x}$ production target heated up to $2000{ }^{\circ} \mathrm{C}$ to obtain low energetic beams $(\sim 30 \mathrm{keV})$ of radioactive isotopes. Photofission products were extracted from the target with a hot plasma universal ion source FEBIAD (Forced Electron Beam Induced Arc Discharge) [24] with 1+ charge state and delivered to the mass separator PARRNe. The resolution $(\mathrm{m} / \delta \mathrm{m} \approx 1500)$ was high enough to provide isobaric selection of $\mathrm{A}=123$. The members of the isobaric chain such as Pd and Rh were refractory metals whose efficiency to release from the source was very low.

The modular detection system equipped with the BEDO tape station [21] was installed behind the mass separator PARRNe and was operated in the neutron detection configuration. It consisted of highly efficient neutron detector TETRA [22]; a $\sim 4 \pi \beta$ scintillator and a High-Purity coaxial Germanium $\gamma$-ray detector (EUROGAM-1 type [25]). Massseparated beams were collected onto an Al-coated Mylar movable tape at the geometrical centre of the array. TETRA is one of the long-neutron counters constructed at JINR Dubna originally for spontaneous fission studies [26] but modified to allow $\beta$-delayed neutron measurements. TETRA response to the $\beta$-delayed neutron is fully understood by means of MCNP simulations [27] and by the reference measurements of $\beta$ decay of heavy ${ }^{96,97} \mathrm{Rb}$ isotopes [28]. The neutron efficiency $\epsilon_{n}=53(2) \%$ was measured with a ${ }^{252} \mathrm{Cf}$ source. The Ge(HP) detector was calibrated using standard sources of ${ }^{60} \mathrm{Co}$ and ${ }^{152} \mathrm{Eu}$. The absolute efficiency measured for $1.3 \mathrm{MeV} \gamma$-rays was $\epsilon_{\gamma} \sim 0.6 \%$. The efficiency of the $\beta$ detector $\epsilon_{\beta}=37(4) \%$ was determined using ratios of area under peaks for identified transitions in $\beta$-decay daughters in the direct $\gamma$ and $\beta$ gated $\gamma$-spectra applying the coincidence method described in Refs. [22,28].

The measurement cycle was divided into three stages. The data was collected for $\mathrm{T}_{\text {meas }}=\mathrm{T}_{\text {beam }}+\mathrm{T}_{\text {decay }}$ : after the accumulation of a source during $\mathrm{T}_{\text {beam }}$, the beam was deviated 
Table 1 Tape-cycle parameters in ms used for the A $=123$ settings: $\mathrm{T}_{\text {beam }}$ and $\mathrm{T}_{\text {decay }}$ are the duration of the beam collection and the beamoff source decay running acquisition time correspondingly; $\tau_{\text {data }}, \tau_{b g}$ and $\tau_{\text {decay }}$ are as defined in Fig. 1 and $\mathrm{N}_{\text {cycles }}$ is the total number of tape cycles

\begin{tabular}{llllll}
\hline $\mathrm{T}_{\text {beam }}$ & $\mathrm{T}_{\text {decay }}$ & $\tau_{\text {data }}$ & $\tau_{\text {decay }}$ & $\tau_{\text {bg }}$ & $\mathrm{N}_{\text {cycles }}$ \\
\hline 1000 & 4000 & 1500 & 500 & 1500 & 63 \\
\hline
\end{tabular}

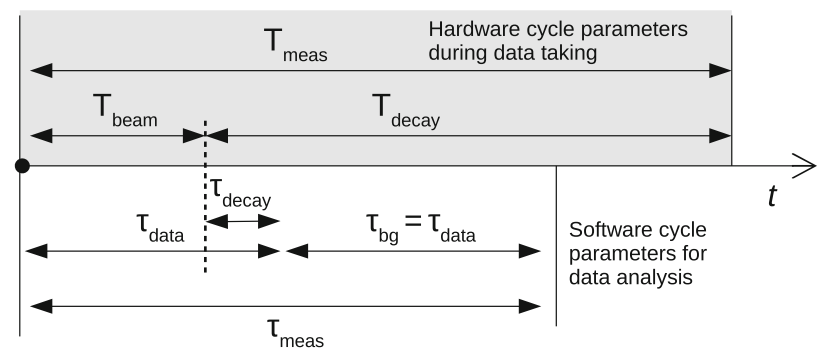

Fig. 1 Time-cycling parameters for the data taking and analysis

to allow counting the decay of the source for $\mathrm{T}_{\text {decay }}$. At the third stage the data acquisition system was stopped and the tape was rewound for $2 \mathrm{~m}$ to transport the source, accumulated on the tape, outside the detection system. Adjusting the $\mathrm{T}_{\text {beam }}$ and $\mathrm{T}_{\text {decay }}$ parameters, see Table 1 , it was possible to favour detection of decay radioactivity from ${ }^{123} \mathrm{Ag}$. Since the half-life of ${ }^{123} \mathrm{Ag}$ is in a hundred $\mathrm{ms}$ range, in the offline analysing procedure for every cycle, only the data collected within $\tau_{\text {meas }}=\tau_{\text {data }}+\tau_{b g}$, defined as in Fig. 1 was sorted. The $\gamma$-ray spectra incremented within $\tau_{d a t a}$ and $\tau_{b g}$ were denoted as $\mathrm{S}_{\text {data }}$ and $\mathrm{S}_{b g}$ correspondingly.

Figure 2 (top), displays the $\beta$ gated $\gamma$-spectrum $\left(\mathrm{S}_{\text {data }}^{\beta}\right)$ which reveals transitions characterising $\beta$-decay of the $\mathrm{A}=123$ isobaric multiplet (Ag, Cd, In). Whereas the background spectra $\mathrm{S}_{b g}^{\beta}$, Fig. 2 (middle), shows transitions only in the longer lived In and Sn. The subtraction of both spectra resulted in $S_{s u b}^{\beta}$ spectrum plotted in Fig. 2 (bottom). In this spectrum transitions in ${ }^{123} \mathrm{Cd}$ were favoured to be on the positive part of the scale whereas the $\gamma$-ray transitions in the longer-lived daughters are ,reversed” to the negative part. This method allowed us to clearly observe five transitions previously attributed to ${ }^{123} \mathrm{Cd}$ [18]. The relative intensities measured in both $\mathrm{S}_{d a t a}^{\beta}$ and $\mathrm{S}_{\text {sub }}^{\beta}$ are in agreement with the ones quoted in Ref. [18], see Table 2. The high production rates of $\mathrm{Cd}$ and $\mathrm{In}$ isotopes together with a small $\mathrm{P}_{1 n}$ value for ${ }^{123} \mathrm{Ag}$ as well as the large neutron coincidence window (100 $\mu$ s due to the neutron moderation) resulted in a fact that the neutron tag did not lead to further purification of the recorded $\gamma$-ray spectra.
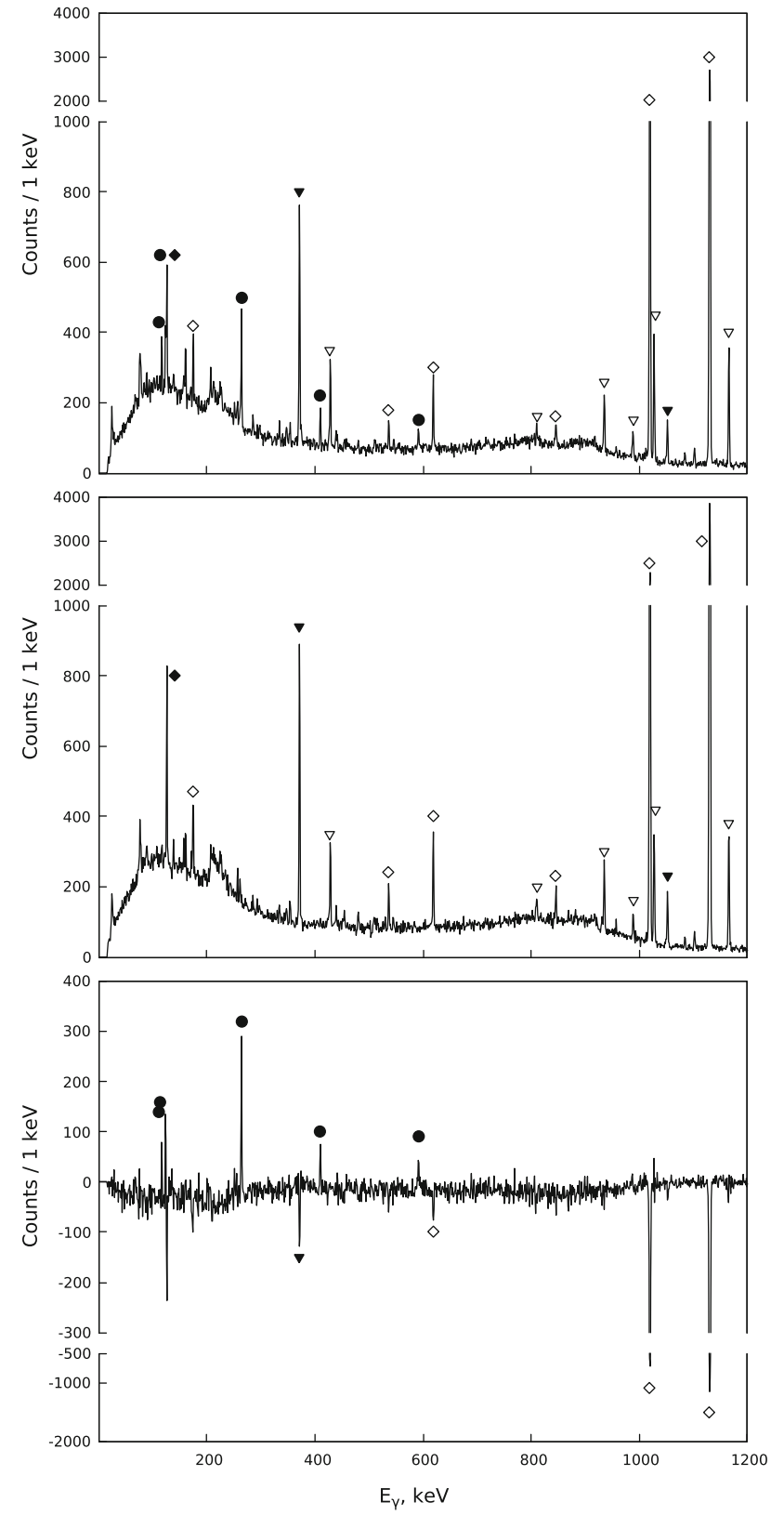

Fig. $2 \beta$ gated $\gamma$-spectra integrated over 63 cycles recorded for the $\mathrm{A}=123$ settings. $\mathrm{S}_{\text {data }}^{\beta}$ (top), $\mathrm{S}_{b g}^{\beta}$ (middle), $\mathrm{S}_{\text {sub }}$ (bottom) see text for details. Transitions are indicated in the daughter nuclei: ${ }^{123} \mathrm{Ag} \rightarrow{ }^{123} \mathrm{Cd}(\bullet) ;{ }^{123} \mathrm{Cd} \rightarrow{ }^{123} \mathrm{In}(\boldsymbol{\nabla}) ;{ }^{123} \mathrm{Cd}^{m} \rightarrow{ }^{123} \mathrm{In}(\nabla)$; ${ }^{123} \mathrm{In} \rightarrow{ }^{123} \mathrm{Sn}(\diamond) ;{ }^{123} \mathrm{In}^{m} \rightarrow{ }^{123} \mathrm{Sn}(\diamond)$

\section{$3 \mathbf{P}_{1 n}$ extraction procedure}

The $\mathrm{P}_{1 n}$ value of a neutron precursor $(\mathrm{A}, \mathrm{Z})$ is the ratio of the number of decays to the $\beta$-delayed neutron daughter $\left(\mathrm{N}_{\beta d n}\right)$ to the total number of $\beta$-decays of the precursor $\left(\mathrm{N}_{\text {total }}\right)$. The experimental methods to derive $\mathrm{P}_{1 n}$ differ in a way how $\mathrm{N}_{\beta d n}$ and $\mathrm{N}_{\text {total }}$ are inferred. In the present measurements $\mathrm{N}_{\beta d n}$ was assumed to be equal to the number of neutrons detected by TETRA and corrected by the background events and the 


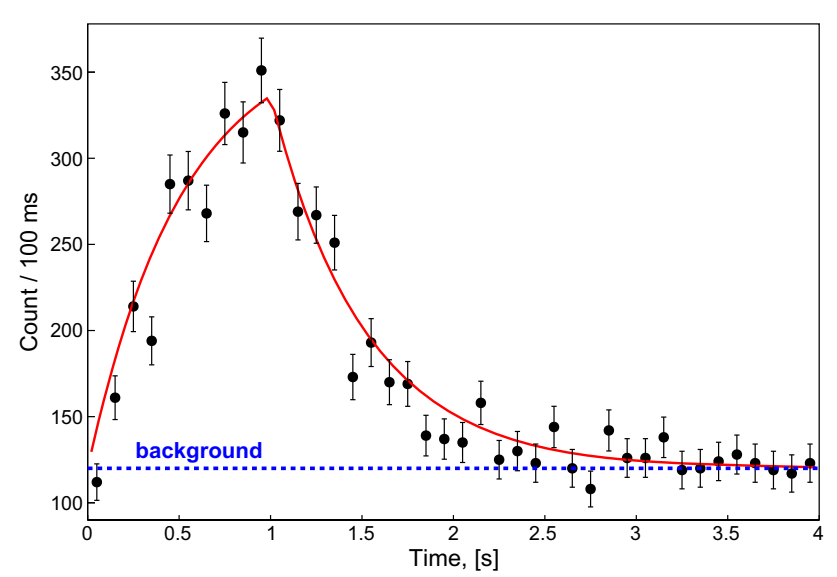

Fig. 3 Neutron activity curve from photo-fission fragments recorded for the $A=123$ mass separator settings. The curve denoted by black points was accumulated within 93 cycles, see Table 1; the red curve is the result of the obtained fit with $\mathrm{T}_{1 / 2}=0.350(20) \mathrm{s}$

neutron deletion efficiency $\epsilon_{n}$. The integrated neutron activity curve accumulated over 63 cycles for the $A=123$ mass separator settings is presented in Fig. 3. The neutron background was of the magnitude of $\approx 20 \mathrm{n} / \mathrm{s}$ thanks to the boron polyethylene shield of TETRA and to the additional separator shielding (see Ref. [22]). Due to a negative $\mathrm{Q}_{\beta n}$ window for other members of the $\mathrm{A}=123$ isobaric multiplet the recorded neutron activity was solely attributed to the $\mathrm{Ag}^{123} \stackrel{\beta-\mathrm{n}}{\longrightarrow} \mathrm{Cd}^{122}$ decay or background activity. The half-life found fitting the neutron activity curve resulted in $0.350(20) \mathrm{s}$.

The total number of ${ }^{123} \mathrm{Ag}$ decays $\left(\mathrm{N}_{\text {total }}\right)$ was derived using the observed $\gamma$-ray transitions, listed in Table 2, and known, from Ref. [18], absolute intensities of $\beta$-decay to the ${ }^{123} \mathrm{Cd}$ excited states. Thus for the $i$-th $\gamma$-ray transition in ${ }^{123} \mathrm{Cd}$ characterized by the $\mathrm{I}_{\beta}^{i}$ transition probability, the $\mathrm{p}_{1 n}^{i}$ can be found as:

$p_{1 n}^{i}=\frac{N_{\beta d n}}{N_{\text {total }}}=\frac{N_{n}}{\epsilon_{n}} \cdot \frac{\epsilon_{\gamma_{i}} \cdot \epsilon_{\beta} \cdot I_{i}^{\beta}}{A_{\gamma^{i}}} \cdot\left(1-T_{\gamma}^{\text {dead }}\right)$,

Table 2 Transitions observed in ${ }^{123} \mathrm{Ag} \stackrel{\beta-}{\longrightarrow}{ }^{123} \mathrm{Cd}$ decay. Relative intensities $\left(\mathrm{I}_{\gamma \beta}^{r e l}\right)$ measured in the $\gamma(\beta)$ full $\left(\mathrm{S}_{\text {data }}^{\beta}\right)$ and subtracted $\left(\mathrm{S}_{\text {sub }}^{\beta}\right)$ spectra; relative $\left(\mathrm{I}_{\text {ref }}^{\text {rel }}\right)$ and absolute $\left(\mathrm{I}_{a b s}^{\beta}\right)$ intensities taken from Ref. [18]

\begin{tabular}{|c|c|c|c|c|}
\hline \multirow[t]{2}{*}{$\mathrm{E}_{\gamma}$} & \multicolumn{2}{|c|}{$\mathrm{I}_{\gamma \beta}^{r e l}, \%$} & \multirow[t]{2}{*}{$\mathrm{I}_{r e f}^{r e l}$} & \multirow[t]{2}{*}{$\mathrm{I}_{a b s}^{\beta}$} \\
\hline & $\mathrm{S}_{\text {sub }}^{\gamma \beta}$ & $\mathrm{S}_{\text {data }}^{\gamma \beta}$ & & \\
\hline $\mathrm{keV}$ & $\%$ & & $\%$ & \\
\hline $116.7(5)$ & $25(3)$ & $27(3)$ & $21(4)$ & $7.6(1)$ \\
\hline $123.9(5)$ & $15(2)$ & n.o. & $16(2)$ & $5.8(4)$ \\
\hline 264.3(5) & $100(8)$ & 100(9) & $100(3)$ & $36(3)$ \\
\hline $410.1(5)$ & $40(5)$ & $54(7)$ & $40(12)$ & $13.2(5)$ \\
\hline $591.8(5)$ & $28(4)$ & $30(6)$ & $29(15)$ & $8.2(3)$ \\
\hline
\end{tabular}

where $\mathrm{A}^{i_{\gamma}}$ is the number of counts under the corresponding peak in the $S_{\text {data }}^{\beta}\left(S_{s u b}^{\beta}\right)$ spectra, $\epsilon_{n}, \epsilon_{\beta}$ and $\epsilon_{\gamma_{i}}$ are efficiencies of neutron, $\beta$ and $\gamma$ (for $i$-th transition) detection respectively and $\mathrm{T}_{\gamma}^{\text {dead }}$ is the dead time of the $\gamma$ electronic channel. The dead time was estimated by monitoring the occupation fraction of the channel. The average dead time over $\tau_{\text {meas }}$ was $\mathrm{T}_{\gamma}^{\text {dead }}=34(6) \%$. The adopted $P_{1 n}$ value then was calculated as the weighted average of $p_{1 n}^{i}$ extracted for different transitions.

This procedure was applied twice to derive the $P_{1 n}$ value using $\mathrm{S}_{d a t a}^{\beta}$ and $\mathrm{S}_{s u b}^{\beta}$ spectra. In case of $\mathrm{S}_{d a t a}^{\beta}$ the $N_{\beta d n}$ was measured as the number of neutrons detected within $\tau_{\text {data }}$ and corrected for the neutron background. In case of $\mathrm{S}_{\text {sub }}^{\beta}$ the $N_{\beta d n}$ was defined as the difference between the number of neutrons detected within $\tau_{d a t a}$ and $\tau_{b g}$. The 1.01(24) $\%$ and 1.03(36) \% values for $\mathrm{P}_{1 n}$, obtained using $\mathrm{S}_{\text {data }}^{\beta}$ and $\mathrm{S}_{\text {sub }}^{\beta}$ spectra respectively, are consistent within the error bars. The adopted values $\mathrm{P}_{1 n}=1.01(24) \%$ (as the most precise) and $\mathrm{T}_{1 / 2}=0.350(20) \mathrm{ms}$ are in good agreement with the results of different experiments cited in Table 3 .

After determining $\mathrm{P}_{1 n}$ and $\mathrm{T}_{1 / 2}$ the ${ }^{123} \mathrm{Ag}$ production yield $(\Phi)$ was estimated to be $\approx 7700 \mathrm{~s}^{-1}$ from the equation:

$$
\int_{0}^{T_{\text {beam }}} A^{\text {beam }}(t)+\int_{T_{\text {beam }}}^{\tau_{\text {decay }}} A^{\text {decay }}(t)=N_{\text {total }}
$$

where $\mathrm{A}^{\text {beam }}(\mathrm{t})$ and $A^{\text {decay }}(\mathrm{t})$ are the activity of ${ }^{123} \mathrm{Ag}$ during the collection and decay measurements respectively defined using the Bateman rules:

$$
\begin{aligned}
& \int_{0}^{T_{\text {beam }}} \Phi \cdot\left(1-e^{-t \cdot \lambda}\right) \delta t+\int_{T_{\text {beam }}}^{\tau_{\text {decay }}} \Phi \cdot e^{-\left(t-T_{\text {beam }}\right) \cdot \lambda}\left(1-e^{-T_{\text {beam }} \cdot \lambda}\right) \delta t \\
& \quad=N_{\text {total }},
\end{aligned}
$$

where $\lambda$ is the decay constant of ${ }^{123} \mathrm{Ag}$.

The production yields of ${ }^{123} \mathrm{Cd},{ }^{123} \mathrm{Cd}^{m}$ and ${ }^{123} \mathrm{In}$, delivered with the beam in the present experiment and reported in Table 4, were estimated by using sets of Bateman equations similar to Eqs. 2 and 3, known half-lives and the absolute intensities of $\beta$-decay to excited states in daughter nuclei cited in the literature [32].

\section{Conclusion}

The paper reports on the first measurement of a delayed neutron precursor in the neighbourhood of ${ }^{132} \mathrm{Sn}$ produced at ALTO facility. $\beta$-decay of the neutron-rich ${ }^{123} \mathrm{Ag}$ was investigated using a $\mathrm{A}=123$ mass-separated beam delivered to the neutron detector TETRA set up at the BEDO tape station. The detector arrangement allowed simultaneous recording of $\beta$-, neutron- and $\gamma$-radiation accompanying the decay of ${ }^{123} \mathrm{Ag}$, for the first time. In addition to the ${ }^{123} \mathrm{Ag}$ ground state, the isomeric and ground states in ${ }^{123} \mathrm{Cd}$ and ${ }^{123}$ In were also populated by induced uranium photofission. The iden- 
Table $3 \mathrm{~T}_{1 / 2}$ and $\mathrm{P}_{1 n}$ of ${ }^{123} \mathrm{Ag}$ neutron precursors. The type of $\beta$ delayed activity for half-life ( $\gamma, \beta$, neutron or i- $\beta-$ ion- $\beta$ correlated decay curve) is shown by the superscript; ${ }^{123} \mathrm{Ag}$ production method is

\begin{tabular}{|c|c|c|c|c|c|}
\hline $\mathrm{T}_{1 / 2}$ & $\begin{array}{l}\mathrm{P}_{n} \\
\mathrm{~ms}\end{array}$ & $\begin{array}{l}\text { Measured } \\
\%\end{array}$ & $\begin{array}{l}\text { Neutron } \\
\text { activities }\end{array}$ & $\begin{array}{l}\text { Production } \\
\text { detector }\end{array}$ & $\begin{array}{l}\text { Facility } \\
\text { method }\end{array}$ \\
\hline $350(20)^{n}$ & $1.01(24)$ & $\gamma, \beta, \mathrm{n}$ & TETRA & mass. sep. & ALTO, present \\
\hline $272(24)^{\beta}$ & $1.0(5)$ & $\beta, \mathrm{n}$, ion & NERO & frag. & MSU, [17] \\
\hline $293(7)^{n}$ & - & $\beta, \mathrm{n}$ & not reported & laser & ISOLDE, [29] \\
\hline $300(10)^{n}$ & $0.55(9)$ & $\beta, \mathrm{n}$ & $40 \times{ }^{3} \mathrm{He}$ & mass. sep. & TRISTAN, [16] \\
\hline $350(40)^{\gamma}$ & - & $\gamma$ & - & mass. sep. & TANDAR [18] \\
\hline $300(20)^{\gamma}$ & - & $\gamma, \beta$ & - & mass. sep. & TRISTAN, [30] \\
\hline $390(30)^{n}$ & - & $\beta, \mathrm{n}$ & $30 \times{ }^{3} \mathrm{He}$ & mass. sep. & OSIRIS $[14,15]$ \\
\hline $0.296(7)$ & $0.56(9)$ & \multicolumn{4}{|c|}{ Recommended by evaluator [31] } \\
\hline
\end{tabular}

indicated as mass. sep. or laser for ISOL mass separated and selectively laser ionized fission fragments correspondingly; or frag. — reaction of fragmentation
Table 4 Measured production yields $(\Phi)$ for $A=123$ mass isotopes implanted into the tape; $\mathrm{P}_{1 n}$ and $\mathrm{T}_{1 / 2}$ for ${ }^{123} \mathrm{Ag}$ as measured in this work, for others as recommended by the evaluator [32]

\begin{tabular}{llll}
\hline & $\mathrm{T}_{1 / 2}, \mathrm{~s}$ & $\mathrm{P}_{1 n}, \%$ & $\Phi, \mathrm{s}^{-1}$ \\
\hline${ }^{123} \mathrm{Ag}$ & $0.350(50)$ & $1.01(24)$ & $7.7(40) \cdot 10^{3}$ \\
${ }^{123} \mathrm{Cd}$ & $2.10(2)$ & - & $\sim 4.7 \cdot 10^{4}$ \\
${ }^{123} \mathrm{Cd}^{m}$ & $1.82(3)$ & - & $\sim 1.4 \cdot 10^{5}$ \\
${ }^{123} \mathrm{In}$ & $6.17(5)$ & - & $\sim 1.4 \cdot 10^{6}$ \\
\hline
\end{tabular}

tification of the isotopes was reliably performed based on the recorded $\beta$ gated $\gamma$-spectra. Due to the fact that neutron emission was not energetically possible in the members of the $\mathrm{A}=123$ isobaric multiplet, other than $\mathrm{Ag}$, the observed neutron activity was attributed to the $\beta$-n decay of ${ }^{123} \mathrm{Ag}$. The half-life measured from the detected neutron activity agreed with values reported from previous experiments. It is worth noting that, in general, $\mathrm{T}_{1 / 2}$ for ${ }^{123} \mathrm{Ag}$ obtained by using neutron activity tends to be slightly longer in comparison to $\beta$ and ion counting techniques. The $\mathrm{P}_{1 n}$ value was found as the ratio of detected neutrons (background subtracted, efficiency corrected) to the total number of ${ }^{123} \mathrm{Ag}$ $\beta$-decays determined from the $\gamma$-rays observed. The adopted $\mathrm{P}_{1 n}$ agreed within the error bars with results of another experiments where $\mathrm{P}_{1 n}$ was derived directly by $\beta$ - and neutroncountings. This agreement provided the indirect proof of the accuracy of the $\beta$-decay intensities to excited states in ${ }^{123} \mathrm{Cd}$ daughter. The results prove the ability of TETRA to be an effective tool using mass-separated beams extracted by the plasma ion source. In the particular case of this experiment, as the yield of Pd was negligibly small, and due to the fact that $\mathrm{Ag}$ was the only neutron precursor on the isobaric chain, the parameters of $\beta$-decay could be still properly measured. Similar techniques will be adopted to study decay of heavier $\mathrm{Ag}$ and $\mathrm{Cd}$ isotopes to be reported in a forthcoming publication.
Acknowledgements The authors wish to acknowledge support from the bilateral agreement JINR-CNRS and the TETRA collaboration. We thank the technical staff of the Tandem/ALTO facility for their assistance with the experiments and for providing excellent quality radioactive beams. The use of one Ge detector from the French-UK IN2P3-STFC Gamma Loan Pool is acknowledged.

Data Availability Statement This manuscript has no associated data or the data will not be deposited. [Authors' comment: All the data are already displayed in the article.]

\section{References}

1. J.S. Al-Khalili, Roeckl Ernst (eds.), The Euroschool Lectures on Physics with Exotic Beams, vol. III (Springer, Berlin, 2009)

2. Y. Blumenfeld, T. Nilsson, P. Van Duppen et al., Phys. Scr. 014023, (2013)

3. B. Pfeiffer, K.-L. Kratz, F.-K. Thielemann, W.B. Walter, Nucl. Phys. A 693, 282 (2001)

4. I.N. Borzov, J.J. Cuenca-García, K. Langanke, G. Martínez-Pinedo, F. Montes, Nucl. Phys. A 814, 159 (2008)

5. M.R. Mumpower et al., Prog. Part. Nuc. Phys. 86, 86 (2016)

6. D. Savran, T. Aumann, A. Zilges, Prog. Part. Nucl. Phys. 70, 210 (2013)

7. N. Paar, D. Vretenar, E. Khan, G. Colò, Rep. Prog. Phys. 70, 691 (2007)

8. M. Arnould, S. Goriely, K. Takahashi, Phys. Rep. 450, 97 (2007)

9. M. Wang, G. Audi, F.G. Kondev, W.J. Huang, S. Naimi, X. Xu, Chin. Phys. C 41, 030003 (2017)

10. A. Gottardo, D. Verney, I. Deloncle et al., Phys. Lett. B 772, 359 (2017)

11. A. P. Severyukhin, N. N. Arsenyev, Yu. E. Penionzhkevich et al., in Proceedings of EXON-2018. World Scientific (2019) p. 345

12. A.P. Severyukhin, N.N. Arsenyev, I.N. Borzov et al., Phys. Rev. C 101, 054309 (2020)

13. M. Arnould, S. Goriely, Prog. Part. Nucl. Phys. 112, 103766 (2020)

14. E. Lund, G. Rudstam, Phys. Rev. C 13, 321 (1976)

15. E. Lund, G. Rudstam, Phys. Rev. C 13, 1544 (1976)

16. P.L. Reeder, R.A. Warner, R.L. Gill, Phys. Rev. C 27, 3002(R) (1983)

17. F. Montes, A. Estrade, P.T. Hosmer et al., Phys. Rev. C 73, 035801 (2006)

18. H. Huck, A. Jech, G. Marti et al., Phys. Rev. C 40, 1384 (1989) 
19. F. Azaiez, S. Essabaa, F. Ibrahim, D. Verney, Nucl. Phys. News 23, 5 (2013)

20. S. Essabaa, N. Barré-Boscher, M. Cheikh Mhamed, Nucl. Instrum. Methods Phys. Res. Sect. B 317, 218 (2013)

21. A. Etile, D. Verney, N.N. Arsenyev et al., Phys. Rev. C 91, 064317 (2015)

22. D. Testov, D. Verney, B. Roussière, Nucl. Inst. Meth. A 815, 96 (2016)

23. D. Verney, D. Testov, F. Ibrahim et al., Phys. Rev. C 95, 054320 (2017)

24. S. Sundell, H. Ravn, Nucl. Phys. B 70, 160 (1992)

25. C. Beausang, S. Forbes, P. Fallon et al., Nucl. Inst. Meth. A 313, 37 (1992)

26. D. Testov, Ch. Briançon, S. Dmitriev et al., J. Phys. Atom. Nucl. 72, 1 (2009)
27. D. Testov, E. Kuznetsova, J.N. Wilson, JINST 10, P09011 (2015)

28. D. Testov, D. Verney, Yu. E. Penionzhkevich, JINST 14, P08002 (2019)

29. V.N. Fedoseyev, Y. Jading, O.C. Jonsson et al., Z. Phys. A 353, 9 (1995)

30. H. Mach, R.L. Gill, D.D. Warner et al., Phys. Rev. C 34, 1117 (1986)

31. J. Liang, B. Singh, E.A. McCutchan, Nucl. Data Sheets 168, 1-116 (2020)

32. S. Ohya, Nucl. Data Sheets 102, 547 (2004) 